Methode von Victor Meyer und Riddle $=1127^{\circ}$ annehmen. Unsere Bestimmungen können also dafür einen Anhalt geben, dass man die für jene empirischen Schmelzpunktproben bisher angenommenen Temperaturen um etwa $100^{\circ}$ erhöhen sollte.

Über die Temperaturen, die beim Brennen der Mergel im Ofen herrschten, können wir Folgendes mittheilen: Wir haben in einem hessischen Tiegel die Seger'schen Kegel auf eine Unterlage aufgestellt, den bedeckten Tiegel in den Ofen eingesetzt und am Schlusse des Brandes ihr Verhalten beobachtet.

Bei der intensivsten Hitze des Ofens, die wir durch einen vollkommenen Luftzutritt erzielten, wurden von den eingesetzten Kegeln die Kegel von No. 8 bis 15 glatt zusammengeschmolzen; der Kegel 15 eine starke und 16 geringe Verkrümmung. Nach Seger wird es einer Temperatur von $1526^{\circ}$ entsprechen (in Wirklichkeit also jedenfalls erheblich mehr, wie wir oben nachgewiesen haben).

In einem bei um die Hälfte vermindertem Luftzutritt angefeuerten Ofen schmolzen die Kegel No. 1, 2, 3, 4 ganz, die übrigen bis No. 10 zeigten nur eine geringe Sinterung. Die Temperatur ist also nach Seger $1237^{\circ}$

Als wir nun die Mergel 10 und 11 der höchsten Temperatur des Ofens ausgesetzt hatten, so bekamen wir vollständig verschlackte Producte. Der Schmelzpunkt dieser Steine wird also unter $1526^{\circ}$ (durch Seger'sche Kegel angezeigt) liegen. Die Angaben, die in den Lehrbüchern angeführt sind, wonach die Temperatur, bei welcher der Portlandcement gebrannt wird, auf $2000^{\circ} \mathrm{zu}$ schätzen ist $\left.{ }^{20}\right)$, finden sich daher nicht bestätigt. Dieneueren Angaben von M. M e y er ${ }^{21}$ ), wonach die Schmelzpunkte verschiedener Cementrohmischungen nicht weit von einander und zwar bei $1450^{\circ}$ (nach Seger'schen Kegeln bestimmt) liegen, werden dagegen richtiger sein. Was die Kegel betrifft, so haben wir die von Dr. Jochum ${ }^{22}$ ) bemerkte Unregelmässigkeit beim Schmelzen nicht beobachtet.
Die Antwort von Seger, worin er diese Unregelmässigkeit im Schmelzen auf die Unregelmässigkeiten des Feuers im Ofen zurückführt, welche durch die Luftströmungen hervorgebracht werden, scheint demnach berechtigt $z u$ sein. Bei uns wurde die Erhitzung der Kegel in einer ruhenden Luftregion vorgenommen.

Technsch-chemisches Laboratorium des eidg. Polytechnikums in Zurich.

Zur titrimetrischen Trennung von Hydroxyden und Carbonaten und Bicarbonaten und Carbonaten der Alkalien, alkalischen Erden und der Magnesia.

$$
\text { Von }
$$

\section{Dr. C. Kippenberger.}

(Aus dem pharmaceutischen Institute und Laboratorium fur angew. Chemie der Universitat Munchen.)

Ich habe unlängst an anderer Stelle ${ }^{1}$ ) dargethan, dass Hämatoxylin, Gallein, Alcannin, Gentianablau, Bleu de Lyon und Bleu de Poirrier mit den Carbonaten und Bicarbonaten der Alkalien, alkalischen Erden und der Magnesia, wie auch mit den jeweiligen einzelnen Carbonaten und Bicarbonaten der genannten Basen verschiedene Farbenerscheinungen geben, und dass letztere nicht allein eine qualitative Unterscheidung der an diese verschiedenen Basen gebundenen Kohlensäure ergeben, sondern auch eine quantitativ verlaufende titrimetrische Trennung von Carbonat und Bicarbonat ermöglichen. Die Farbenerscheinungen der genannten Indicatoren sind bei einzelnen Carbonaten von den durch die Hydroxyde hervorgerufenen verschieden, während die der letzteren untereinander gleich sind. Folgende Zusammenstellung möge die verschiedenartige Reactionsfähigkeit der Indicatoren gegenüber den verschiedenen Carbonaten und Hydroxyden übersichtlich wiedergeben:

\begin{tabular}{|c|c|c|c|c|c|c|c|}
\hline & Durch: & $\mid \begin{array}{c}\text { Ha- } \\
\text { matoxylin }\end{array}$ & Gallein & Alcannin & Gentianablau & $\begin{array}{c}\text { Bleu } \\
\text { de Lyon }\end{array}$ & Bleu de Poirrier \\
\hline Hydroxyd & & blau & blau & blau & dunkelblau & roth & roth \\
\hline Alkali- & $\left\{\begin{array}{l}\text { carbonat } \\
\text { bicarbonat }\end{array}\right.$ & roth \{ & $\begin{array}{l}\text { blau, allmahlich } \\
\text { roth werdend } \\
\text { roth }\end{array}$ & $\begin{array}{l}\text { blau } \\
\text { roth }\end{array}$ & $\begin{array}{l}\text { roth } \\
\text { blau }\end{array}$ & $\begin{array}{l}\text { roth } \\
\text { blau }\end{array}$ & $\begin{array}{l}\text { bei concentr. Los.: roth } \\
- \text { verd. } \\
\text { blau }\end{array}$ \\
\hline Alk. Erd.- & $\left\{\begin{array}{l}\text { carbonat } \\
\text { bicarbunat }\end{array}\right.$ & $\int \operatorname{roth}$ & roth & roth & blau & blau & blan \\
\hline Magnesium- & $\left\{\begin{array}{l}\text { carbonat } \\
\text { bicarbonat }\end{array}\right.$ & $\begin{array}{l}\text { blau } \\
\text { roth }\end{array}$ & $\begin{array}{l}\text { blau } \\
\text { roth }\end{array}$ & $\begin{array}{l}\text { Wlau } \\
\text { roth }\end{array}$ & blau & $\begin{array}{l}\text { roth } \\
\text { blau }\end{array}$ & blau \\
\hline
\end{tabular}

20) Michaelis S. 136.

21) Fischer's Jahresb. 1887, 826.

22) Thonind. 88 , No. 6 .
1) Forschungsber. uber Lebensmittel, Hyg., forens. Chem. I. 1894, Heft 9. 
Titrirt man eine earbonathaltige Lösung mit Säure, so wird bekanntermaassen nach den Gleichungen:

und

$$
\mathrm{Na}_{2} \mathrm{CO}_{3}+\mathrm{HCl}=\mathrm{NaCl}+\mathrm{NaHCO}_{2}
$$

$$
\mathrm{NaHCO}_{3}+\mathrm{HCl}=\mathrm{NaCl}+\mathrm{CO}_{2}+\mathrm{H}_{2} \mathrm{O}
$$

zunächst das Carbonat in Bicarbonat verwandelt. War nun die verschiedenartige Reaction der Farbenerscheinungen der genannten Indicatoren gegenüber den Hydroxyden, Carbonaten und Bicarbonaten eine quantitativ verlaufende, oder präciser ausgedrückt: trat die für Bicarbonat charakteristische Färbung erst nach der vollständigen Umwandlung von Carbonat in Bicarbonat ein, so lag es nahe, die Indicatoren zur quantitativen Bestimmung der Hydroxyde neben Carbonaten zu verwerthen. Und in der That ergaben die Versuche Resultate, welche zur Aufstellung von Titrationsmethoden berechtigen. Die Titration erfolgte da, wo die Flüssigkeit ein geringes Volumen einnahm, in einem mit Gummistopfen fest verschliessbaren Reagenscylinder oder in einem engen Cylinder mit Glasstöpsel, und dort, wo diese durch Vermebrung des Volumens der Flüssigkeit nicht ausreichten, in einer mit Glasstöpsel versehenen Flasche entsprechender Grösse unter langsamem Zusatz von Säure, wodurch ein Verlust an Kohlensäure möglichst vermieden wurde. Indem sich nun Methylorange mit den meisten Indicatoren vermischen lässt, ohne dass dadurch die Reactionsfähigkeit dieses Indicators gegenüber der Säure an Schärfe einbüsst, lässt sich die Bestimmung der kohlensauren Verbindungen neben Hydroxyden und auch die der Bicarbonate neben den Carbonaten in einer Operation ausfübren.

Anwendung von Hämatoxylin. Benutzt wurde eine möglichst neutral eingestellte Lösung von Hämatoxylin in 50 proc. Alkohol. Da Hämatoxylin mit Alkalicarbonat und -bicarbonat rothe Farbenerscheinung gibt (mit Bicarbonat dunkelroth), so lässt sich dasselbe zur Bestimmung beider Verbindungen neben einander nicht benutzen, wohl aber lässt sich eine Bestimmung von Magnesiumearbonat neben Alkalicarbonat bewirken, da Magnesiumcarbonat mit dem Indicator eine charakteristische Blaufärbung zeigt, auch wenn Alkalicarbonat in nicht allzugrosser Menge zugegen ist.

$100 \mathrm{cc}$ einer Magnesiumcarbonatlösung, die nach genauer Titrations- und Gewichtsanalyse $0,001239 \quad \mathrm{MgCO}_{3}$ entbielten, zur Sättigung also 29,5 cc $1 / 100 \mathrm{~N}$.-Säure verbrauchten, ergaben nach Zusatz einiger Tropfen der Hämatoxylinlösung von blau bis zur purpurvioletten $\mathrm{F}$ ärbung titrirt $(=\mathrm{Bi}$ - carbonatbildung): je 10,8 und $10,2 \mathrm{cc} 1 / 100^{-}$ $\mathrm{N}$. $-\mathrm{H}_{2} \mathrm{SO}_{4}$.

Wurde ein Zusatz von beliebigen Mengen Baryumcarbonatlösung gemacht, so waren nöthig: je $10,6,10,510,1 \mathrm{cc} 1 / 100 \mathrm{~N}$. $-\mathrm{H}_{2} \mathrm{SO}_{4}$ und nach Zusatz von $10 \mathrm{ce} 1 / 10$ Natriumcarbonatlösung wurden verbraucht: je 11,9, $11,6,11,2$ cc $1 / 100$ N.- $\mathrm{H}_{2} \mathrm{SO}_{4}$.

Die Resultate differiren um etwa ein Drittel als Minus von der theoretisch berechneten Menge Säure $\left(=14,75 \mathrm{cc} 1 / 100 \mathrm{~N} .-\mathrm{H}_{2} \mathrm{SO}_{4}\right)$ und es lässt sich dies dadurch erklären, dass das sich hierbei bildende Magnesiumbicarbonat in Sesquicarbonat und freie Kohlensäure zerfällt, eine Thatsache, die ich an der bereits oben citirten Stelle ${ }^{2}$ ) mit analytischen Belegen ergänzt habe und die sich auch bei der nachfolgenden Anwendung des Gallein als Indicator nochmals zeigen wird. Das Magnesiumsesquicarbonat selbst gibt mit Hämatoxylin noch blaue Färbung.

Die Beispiele zeigen aber auch, dass Magnesiumcarbonat vor dem Alkalicarbonat in Bicarbonat oder richtiger in Sesquicarbonat umgewandelt wird, und erst dann, wenn sich die Sesquicarbonatbildung bei Magnesium vollzogen hat, wird auch Alkalicarbonat zunächst in Sesquicarbonat umgewandelt werden, was aus dem merklichen Mehrverbrauch an Säure gegenüber dem bei der Titration mit der reinen Magnesiumcarbonatlösung ersichtlich ist. Eine Sesquicarbonatverbindung der alkalischen Erden scheint es aber nicht zu geben, denn es müsste sonst auch hier der Verbrauch an Säure ein erhöhter gewesen sein.

Setzt man einer Mischung von Alkalihydroxyd und -carbonat Hämatoxylin hin$z u$, so tritt nicht etwa eine das Hydroxyd charakterisirende Blaufärbung ein, sondern es entsteht eine Mischfarbe, etwa dunkelklatschroth. Ist dann nach langsamem Säurezusatz das Hydroxyd durch eine äquivalente Menge Säure in Neutralsalz umgewandelt, so erscheint die das Alkalicarbonat charakterisirende Rothfärbung.

Anwendung von Gallein. Zur Verwendung gelangte eine 1 proc. alkoholische, möglichst neutral eingestellte Galleinlösung. Gallein eignet sich zur Titration von Lösungen der Carbonate der Alkalien und der der Magnesia bedeutend besser als, Hämatoxylin. Die Titration geschieht von der blauen bis zur hellrothen Färbung.

Setzt man einer Mischung von Alkalibydroxyd- und -carbonatlösung Gallein

2) Kritische Studien uher die Methoden der Kohlensäurebestimmung in Trink- und Mineralwässeru. Forschungsber. über Lebensm., Hrg., forens. Chen. I. 1894 . 
100 ce Magnesiumcarbonatlosung (Zus. wie oben) verbrauchten: $12,3 \mathrm{cc}^{1^{\prime}}{ }_{100} \mathrm{~N}_{-}-\mathrm{H}_{2} \mathrm{SO}_{4} \quad 14,75 \mathrm{cc}$

5 - 1/\% Natriumcarbonatlosung

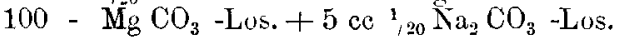

hinzu, so gibt dieses zwar die charakteristische Blaufärbung, aber die Rothfärbung tritt nicht erst nach Zusatz einer zur Bildung von Hydroxyd in Neutralsalz und Umwandlung von Carbonat in Bicarbonat berechneten Menge Säure ein, sondern bereits früher und es ist anzunehmen, dass die Sesquicarbonatbildung hier ebenfalls einen Einfluss auf die Farbenveränderung des Gallein ausübt, jedoch mit dem vor Hämatoxylin hervortretenden Unterschiede, dass bereits das Sesquicarbonat und nicht erst die über Sesquicarbonat hinausgehende kohlensaure Verbindung (wie dies bei Anwendung von Hämatoxylin der Fall ist) die Veränderung hervorruft. Setzt man z. B. zu $10 \mathrm{cc} 1 / 10 \mathrm{~N}$--Natriumcarbonatlösung einige Tropfen Gallein hinzu, so tritt eine Blaufärbung ein, welche schon nach Zusatz von 2,4 cc $1 / 10 \mathrm{~N}$.-Schwefelsäure in eine deutlich erkennbare Rothfärbung umschlägt; mischt man nun 5 ec N.-Natriumhydroxydlösung hinzu, so tritt wieder Blaufärbung ein und nach 1,5 cc $1 / 10 \mathrm{~N}$.-Schwefelsäurezusatz ist die Mischung bereits abermals roth. Hier hatte sich aus der Natriumcarbonatlösung durch den Säurezusatz zunächst nicht etwa Natriumbicarbonat neben Carbonat gebildet, sondern sämmtliches Carbonat war in Sesquicarbonat verwandelt (= Rothfärbung); nach Zusatz von Natriumbydroxyd trat die als Sesquicarbonat gebundene Kohlensäure mit dem Hydroxyd in Wirkung unter Bildung von Natriumcarbonat (= Blaufärbung), worauf nach entsprechendem Säurezusatz wieder die Rothfärbung des Sesquicarbonates eintreten musste. Nebenbei ist dann noch zu berücksichtigen, dass bei längerer Einwirkung von Alkalicarbonatlösung auf Gallein die zuerst eingetretene Blaufärbung in Roth umzuschlagen pflegt (je nach Concentration nach 5-15 Minuten), daher ein entsprechend schnelles Arbeiten nothwendig ist, wodurch kleine Fehler durch Kohlensäureverlust nur selten vermieden werden.

Es ergibt sich nach den theoretischen Betrachtungen von selbst, dass bei Mischungen von Hydroxyden und Carbonaten durch Säurezusatz zunächst Hydroxyd zu Neutralsalz und dann erst das Carbonat in Bicarbonat verwandelt werden wird, denn wäre dies nicht der Fall, so müsste ja Bicarbonat neben Hydroxyd bestehen und dies kann nicht sein, da die mehr als zu Monocarbonat gebundene Koblensäure dann mit dem Hydroxyd Ch. 94 . zur Bildung von Carbonat in Wirkung treten wird. Die oben erwähnten Beobachtungen mit Hämatoxylin ergänzen diese Thatsache, und die mit Gallein gefundenen Farbenumschläge beweisen ferner, dass auch nicht Bicarbonat neben Carbonat bestehen wird, sondern dass sich alsdann Sesquicarbonat bilden wird und erst von dem Momente an, wo sämmtliches Monocarbonat in Sesquicarbonat verwandelt sein wird, tritt Bildung von Bicarbonat ein. Wohl lässt sich die Trennung von Bicarbonat und Carbonat durch Titration unter Anwendung von Gallein von blau- bis zur hellrothen Färbung bewirken, der Übergang ist aber nur für den mit diesem Indicator sehr Geübten annähernd genau fixirt, und es erscheint, dass die Resultate desto besser ausfallen, je verdünnter die Titrationssäure ist (natürlich bis zu einem gewissen Grade; am besten eignet sich solche von $1 / 100$ N.-Stärke). Es wird dadurch eine momentan zu heftige Kohlensäureentwicklung vermieden, welche auf Gallein störend einwirkt. Setzt man alsdann einen Tropfen Methylorangelösung ( 0,5 proc. Lösung in Wasser) zu der Mischung und titrirt mit Säure bis zur beginnenden Rosafärbung, ohne Rücksicht zu nehmen auf die dunklere Mischfarbe, welche nach kurzem Säurezusatz entsteht, so sind sämmtliche Carbonate mit Säure neutralisirt.

10 cc $1 / 10$ N.-Natriumcarbonatlösung verbrauchten so bis zu der durch den Farbenumschlag deutlich erkennbaren Bicarbonatbildung: je 5,2 bis 5,35 cc $1 / 10$ N.-Schwefelsäure und nach Zusatz von 1 bis 2 Tropfen Methylorangelösung insgesammt: je 10,05 cc $1 /{ }_{10}$ N.-Schwefelsäure.

Bei der Titration von 1 cc $1 / 10 \mathrm{~N}$.-Natriumcarbonatlösung +25 cc Wasser mit ${ }^{1 / 100}$ N. - Schwefelsäure war der Farbenumschlag schon bedeutend wahrnehmbarer; es wurden hier verbraucht je: 5,15 und $5,10 \mathrm{cc}$ $1 / 100$ N.-Schwefelsäure und nach Zusatz von Methylorange genau $10 \mathrm{cc}$, also eine die Gesammtmenge Kohlensäure genau anzeigende Menge Säureflüssigkeit. -

Eine in jeder Hinsicht brauchbare und bequeme Verwerthung für die titrimetrische Bestimmung von Hydroxyden neben Carbonaten und Carbonaten neben Bicarbonaten können aber nur diejenigen Indicatoren beanspruchen, welche gegen Sesquicarbonat genau so wie gegen Carbonat reagiren und erst mit Bicarbonat Farbenumschläge ergeben, und dies ist bei der 
Anwendung von Phenolphtalein, Alcannin und Bleu de Lyon der Fall. Zur Verwendung gelangten:

eine 1 proc. alkoholische Lösung von Phenolphtalein bez. Bleu de Lyon und eine gesättigte, möglichst genau eingestellte alkoholische Alcanninlösung (aus dem extractförmigen Präparat bereitet).
Anwendung von Gentianablau und Bleu de Poirrier $\left(\mathrm{C}_{4} \mathrm{~B}\right)$, sowie die ron Indigschwefelsäure und Flavescin. Gentianablau, welches mit Alkalicarbonat rothe Färbung ergibt, steht an Intensität dieser Reactionserscheinung der bei denen bei oben angegebenen Indicatoren sehr nach, und erst in Lösungen von der Stärke der Normal-

\begin{tabular}{|c|c|c|c|c|}
\hline & $\begin{array}{c}\text { Phe- } \\
\text { nolphtalein }\end{array}$ & Alcannin & Bleu de Lyon & ieoretisch \\
\hline & roth farblos & blau & roth & \\
\hline $\begin{array}{c}100 \mathrm{ce} \mathrm{Mg} \mathrm{CO}_{3} \text {-Lös. (wie oben) }=\text { cc } 1 / 100 \\
\text { (Carbonat in Bicarbonat), }\end{array}$ & 14,6 & $\begin{array}{c}15,2 ; 15,5 \\
15,7\end{array}$ & $\begin{array}{l}\text { schon nach } \mathrm{Zu}- \\
\text { satz von } 7,1 \\
\text { roth }\end{array}$ & 14,75 \\
\hline $\begin{array}{c}\left.\text { sodann mit Methylorange bis rosa }=\mathrm{cc}{ }^{1 / 100} \mathrm{~N}^{-}-\mathrm{H}_{2} \mathrm{SO}_{4}\right) \\
\text { (Neutralisationspunkt) in } \mathrm{Sa} .\end{array}$ & 29,3 & 29,55 & 29,5 & 29,50 \\
\hline $\begin{array}{r}10 \mathrm{ccc} 1 / 10 \mathrm{~N}-\mathrm{Na}_{2} \mathrm{CO}_{3}=\text { ec } 1 / 100 \mathrm{~N}-\mathrm{H}_{2} \mathrm{SO}_{4} \\
\text { (Carbonat in Bicarbonat) }\end{array}$ & 4,$9 ; \quad 5,0$ & 5,$0 ; \quad 5,0$ & 5,$0 ; \quad 5,0$ & 5,0 \\
\hline nach Zusatz ron Methylorange bis rosa $=\mathrm{ce}^{1 / 100} \mathrm{~N}_{-}-\mathrm{H}_{2} \mathrm{SO}_{4}$ & 10,$0 ; 10,0$ & 10,$0 ; 10,0$ & 10,$0 ; \quad 10,0$ & 10,0 \\
\hline $\begin{array}{l}1 \mathrm{cc}{ }^{1 / 10} \mathrm{~N} .-\mathrm{Na}_{2} \mathrm{CO}_{3}+25 \mathrm{cc} \mathrm{H}_{2} \mathrm{O}=\mathrm{cc}{ }^{1 / 100} \mathrm{~N}-\mathrm{H}_{2} \mathrm{SO}_{4} \\
\text { nach Zusatz von Methylorange bis rosa }=\mathrm{cc} 1 / 100 \mathrm{~N}^{1}-\mathrm{H}_{2} \mathrm{SO}_{4}\end{array}$ & $\begin{array}{c}4,75 ; 4,85 \\
10,0\end{array}$ & $\begin{array}{l}5,0 ; \quad 5,0 \\
10,0\end{array}$ & $\begin{array}{l}4,95 ; \quad 5,0 \\
10,0\end{array}$ & $\begin{array}{r}5,0 \\
10,0\end{array}$ \\
\hline $\begin{array}{r}5 \mathrm{ec}^{1 / 10} \mathrm{~N} \cdot-\mathrm{KOH}+10 \mathrm{cc}{ }^{1 / 10} \mathrm{~N} \cdot-\mathrm{Na}_{2} \mathrm{CO}_{3}=\mathrm{cc}^{1 / 10}{ }_{10} \mathrm{~N} \cdot-\mathrm{H}_{3} \mathrm{SO}_{4} \\
\text { (Hydroxyd in Neutralsalz und Carbonat in Bicarbonat) }\end{array}$ & 10,$0 ; \quad 9,9$ & 10,$0 ; 10,0$ & 10,$0 ; \quad 10,0$ & 10,0 \\
\hline nach Zusatz von Methylorange bis rosa $=\mathrm{ec}^{1 / 1} / 10 \mathrm{~N}^{-}-\mathrm{H}_{2} \mathrm{SO}_{4}$ & 15,0 & 15,0 & 15,0 & 15,0 \\
\hline $\begin{array}{c}0,5 \mathrm{ce} 1 / 10 \mathrm{~N} . \mathrm{KOH}+0,5 \mathrm{ce} 1 / 10 \mathrm{~N}_{-}-\mathrm{Na}_{2} \mathrm{CO}_{3}+25 \mathrm{cc} \mathrm{H}_{2} \mathrm{O} \\
=\mathrm{ec}^{1 / 100} \mathrm{~N}_{-}-\mathrm{H}_{2} \mathrm{SO}_{4}\end{array}$ & 7,4 & 7,5 & 7,5 & 7,5 \\
\hline nach Zusatz von Methylorange bis rosa $=\mathrm{cc} 1 / 100 \mathrm{~N} \cdot-\mathrm{H}_{2} \mathrm{SO}_{4}$ & 10,0 & 10,0 & 10,0 & 10,0 \\
\hline 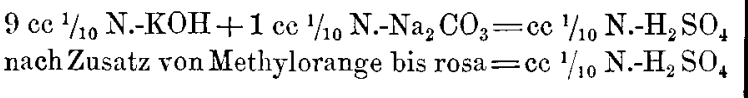 & $\begin{array}{r}9,5 \\
10,0\end{array}$ & $\begin{array}{r}9, \tilde{5} \\
10,0\end{array}$ & $\begin{array}{r}9,5 \\
10,0\end{array}$ & $\begin{array}{r}9,5 \\
10,0\end{array}$ \\
\hline $\begin{array}{l}9 \mathrm{cc}^{1 /} /{ }_{10} \mathrm{~N} .-\mathrm{Na}_{2} \mathrm{CO}_{3}+1 \mathrm{cc}^{1 / 10} \mathrm{~N} .-\mathrm{KOH}=\mathrm{cc} 1 / 10 \mathrm{~N} .-\mathrm{H}_{2} \mathrm{SO}_{4} \\
\text { nach Zusatz von Methylorange bis rosa }=\mathrm{ce}^{1 / 10} \mathrm{~N}_{10}-\mathrm{H}_{2} \mathrm{SO}_{4}\end{array}$ & $\begin{array}{r}5,4 \\
10,0\end{array}$ & $\begin{array}{r}5,5 \\
10,0\end{array}$ & $\begin{array}{r}5,5 \\
10,0\end{array}$ & $\begin{array}{r}5,5 \\
10,0\end{array}$ \\
\hline
\end{tabular}

Die erhaltenen Zahlen ergeben, dass Bleu de Lyon gegenüber Magnesiumcarbonat nicht scharf genug reagirt, um zur quantitativen Bestimmung desselben benutzt werden zu können, und dass Alcannin mit Magnesiumcarbonat um ein geringes $z u$ hohe Resultate ergibt, was letzteres wiederum auf die leichte Dissociationsfähigkeit des Magnesiumbicarbonates zurückzufübren sein wird. Alcannin ist gegen freie Kohlensäure wenig empfindlich und daher ist bei der Titration der Magnesiumcarbonatlösung ein Plus an Kohlensäure und somit auch ein Plus an Titrationssäure die nothwendige Folge. Die Titrationen lassen aber fernerhin erkennen, dass alle drei Indicatoren durch ihre verschiedene Reactionsfähigkeit den Unterschied von Bicarbonaten und Carbonaten wie den von Hydroxyden und Carbonaten der Alkalien scharf zu erkennen geben, selbst dann, wenn sehr verdünnte Lösungen, wie solche in der Stärke von 250. Normal, angewandt werden. Alcannin, Bleu de Lyon, und vornehmlich das erstere, geben im Vergleiche mit Phenolphtalein schärfere Reactionen als dieses, voraussichtlich deshalb, weil Phenolphtalein gegen freie Kohlensäure empfindlicher ist als jene. flüssigkeiten würde die Brauchbarkeit dieses Indicators in Betracht zu ziehen sein.

Bleu de Poirrier, $\mathrm{C}_{4} \mathrm{~B}$, ein von Engel und Ville zur titrimetrischen Bestimmung von Alkalihydroxyd neben -carbonat empfohlener Farbstoff ${ }^{3}$ ), gibt nach Angabe dieser mitAlkalicarbonat blaue, mitHydroxyd rothe Färbung. Werden nun hydroxyd- und carbonathaltige Lösungen mit Säure bis zur blauen Farbe unter den von den Autoren angegebenen Cautelen - 10 bis 20 cc Flüssigkeit, langsames Zufliessenlassen der Säure, um locale Kohlensäureentwicklung zu verhüten - titrirt, so ist Hydroxyd neutralisirt und die Gesammtmenge Kohlensäure lässt sich dann in einem Apparat, wie solcher zur Ammoniakbestimmung üblich ist, durch Versetzen mit Säure, Auffangen der Kohlensäure in Kalilauge und Zurücktitriren des überschüssigen Hydroxydes bestimmen. (Z. anal. 1888,38 .) Bicarbonate neben Carbonaten, sowie auch freie Koblensäure neben Bicarbonaten werden durch Zufügen von Kalilauge und Zurücktitriren des überschüssigen Hydro-

3) Compt. r. 100, 1073; Bull. soc. chim. 44, 1885,17 . 
xyd ebenfalls unter Benutzung dieses Indicators bestimmt. - Die ungünstige Kritik Lunge's (Berl. Ber. 1885, 3291), nach welcher bei der Bestimmung von Hydroxyden neben Carbonaten beständig zu niedrige Resultate erzielt werden, veranlassten Engel und Ville zur Feststellung der angegebenen Cautelen. Als ich nun das Poirrier'sche Blau zur Titration der Carbonate benutzen wollte, fand ich, dass concentrirte Alkalicarbonatlösungen mit demselben roth gefärbt werden und erst, sobald die Concentration der Lösung der Stärke von Normalflüssigkeit gleicbkam, trat blaue Färbung ein, dabei war aber bei durchscheinendem Lichte ein ganz deutlicher Stich in's Röthliche wahrzunehmen. Wurde die Farbe der Flüssigkeit auf weis ser Unterlage betrachtet, so erschien sie rein blau. Lösungen in der Stärke der ${ }^{1 / 10}$ N.-Flüssigkeit dagegen waren sowohl bei auffallendem, wie durchscheinendem Lichte charakteristisch blau gefärbt, ähnlich der Farbe derFehling'schen Mischung. Sesquicarbonat- und Bicarbonatlösungen endlich zeigten, wie vorauszusehen war, deutlich hellere Blaufärbung, da der Indicator, wie dies Engel und Ville bereits angegeben haben, gegen Säure sebr empfindlich ist. Aber auch durch Wasserzusatz wird der Indicator beeinflusst und schlägt dabei leicht in Roth über, so dass er sich bei Feststellung der Carbonate in ganz verdünnten Flüssigkeiten - verdünnter als $1 / 10$ Normal - ebenfalls nicht eignen würde. Die Ausführung des Vorschlags nach Göbel (Chemzg. 1889, 696) Mischungen von Alkalihydroxyden mit -carbonaten mit Säure zunächst unter Benutzung von Phenolphtalein bis zum Eintritt der Farblosigkeit zu versetzen (= Sättigung des Hydroxydes und Cmwandlung des Carbonats in Bicarbonat), und hierauf nach Zusatz einiger Tropfen Bleu de Poirrier von der hellblauen bis zur dunkelblauen Farbe zu titriren, wobei die Differenz zwischen farblos (bez. hellblau) und dunkelblau die Hälfte des vorhandenen Arlkalicarbonates und diese Zahl dann von dem Säurenverbrauch der ersten Titration abgerechnet die dem Hydroxyd äquivalente Menge Säure anzeigen würde, lässt, wie der Versuch zeigt, an Deutlichkeitdes Farbenumschlags zu wünschen übrig. Bessere Resultate erzielte ich, indem ich der mit Bleu de Poirrier versetzten Flüssigkeit nach Titration von der rothen bis zur blauen Farbe (= Neutralisation des Hydroxyds) einige Tropfen Methylorange zusetzte und nun mit Säure weiter titrirte. Zunächst tritt eine missfarbige, grünlichbräunliche Färbung der Flüssigkeit ein, beim Zufügen der Säure sieht man dann dort, wo dieselbe eintröpfelt, lila gefärbte Streifen und genau im Neutralisationspunkte schlägt die inzwischen mehr grünlich gewordene Farbe in charakteristisch Blau über, jedoch nur dann, wenn die angewandte Menge des Bleu de Poirrier die des Methylorange überwiegt. Fügt man hingegen etwas mehr Methylorange, oder auch nur eine dem Zusatz von Bleu de Poirrier gleichkommende Menge Methylorange hinzu, so tritt die den geringsten Überschuss von Säure scharf charakterisirende Rosafärbung des Methylorange hervor, und dieser Farbenumschlag erscbeint noch deutlicber als der durch Bleu de Poirrier unter Benutzung von nur wenig Methylorange beobachtete. Wie aus alledem ersichtlich, dürfte die Anwendung von Bleu de Poirrier für die Praxis wenig geeignet sein, da Concentration der Carbonatlösung das Resultat allzusehr beeinflusst. Bei verdünnten Hydroxydlösungen habe ich, ebenso wie Lunge, beständig zu niedere Resultate erbalten; so trat z. B. in einer Mischung von 5 cc $1 / 10$ N.-Kalilauge $+10 \mathrm{cc}^{1 / 10}$ N.-Natriumcarbonatlösung schon ein deutlicher Umschlag von Roth in Blau ein, als erst 5 cc $1 / 10$ N.-Schwefelsäure verbraucht waren, wäbrend nach der Theorie erst nach 10 ce Säurezusatz eine Blaufärbung hätte eintreten müssen.

Noch weit ungenauer fallen die Resultate bei Anwendung von Indigblauschwefelsäure aus, welche ebenfalls von Engel und Ville zur titrimetriscben Bestimmung von Hydroxyden neben Carbonaten empfohlen wurde (C. r. 100, 1074).

F. Lux empfahl den von ihm durch eine besondere Destillationsart aus Eichenholz gewonnenen und gereinigten Indicator, Flavescin genannt, zurtitrimetrischen Trennung von Alkalihydroxyd und -carbonat, wie auch zur Bestimmung von Bicarbonaten neben Carbonaten (Z. anal. 1880, 457). Auf Zusatz von Alkalicarbonat zu Flavescinlösungen tritt Gelbfärbung ein und das kohlensaure Alkali wird dabei in Flavescin und doppeltkohlensaures Salz zerlegt. Alkalibicarbonat hingegen wird von Flavescin nicht zerlegt und daher auch die Verbindungen von Flavescin mit Alkalien durch überschüssige Kohlensäure in doppeltkohlensaure Salze und Flavescin übergeführt, wobei die durch Alkali hervorgerufene Gelbfärbung, verschwindet und Entfärbung des Flavescin eintritt. L. ergänzt diese Angaben an der Hand einer Reibe von Analysenresultaten und lässt zur Trennung von Hydroxyden und Carbonaten unter Beobachtung derbekannten Vorsichtsmaassregeln - Arbeiten in der Kälte, sanftes Umrühren zur Vermeidung von Kohlensäureverlust zu der mit Flavescin versetzten und dadurch 
gelb gefärbten Flüssigkeit Säure bis zur Entfärbung zuflessen, worauf mit Alkali zurücktitrirt wird, wodurch Hydroxyd + der Hälfte des als Carbonat vorhandenen Alkalis angezeigt wird. Alsdann wird mit überschüssiger Säure versetzt, die Kohlensäure durch Kochen vertrieben und mit Alkali abermals bis zur gelben Farbe zurücktitrirt. Hier ergibt sich dann die Hälfte des vorhandenen Carbonats und daraus und aus der ersten Titration in bekannter Weise das freie Alkali. Zur Trennung von Bicarbonat und Carbonat wird die mit Säure im Überschuss versetzte, also entfärbte Flüssigkeit zunächst mit Alkali auf Gelb zurücktitrirt, wodurch die Hälfte des Carbonats unter Bildung von Bicarbonat aus der anderen Hälfte neutralisirt wird. Durch nunmehrigen Säurezusatz im Überschuss und Vertreiben der Kohlensäure durch Kochen wird das ursprünglich vorhandene oder aus dem Monocarbonat gebildete Bicarbonat zersetzt und der Säureüberschuss kann mit Alkali zurücktitrirt werden. Das Verfahren liefert bei exacter Durchführung recht brauchbare Resultate, ist aber durch die Nothwendigkeit des Kochens der Flüssigkeit, um die freie Kohlensäure zu vertreiben, etwas umständlich. Ich habe gefunden, dass Methylorange auch durch Flavescin nicht beeinflusst wird und habe bei Benutzung dieses Indicators in je der zweiten Phase beider angegebenen Untersuchungsgänge sehr genaue Resultate erzielt und vereinfacht sich die Methode dadurch wesentlich. Der Zusatz von Methylorange geschieht natürlich erst dann, wenn in je der ersten Phase der Untersuchungen mit Alkali auf Gelb zurücktitrirt worden ist.

Bei den oben beschriebenen Metboden veranlasste der jeweilige Zusatz des Indicators mit den Hydroxyden und kohlensauren Salzen Farbenveränderungen, welche die Trennung beider durch einfache Titrationen ermöglichten. Die Methoden der quantitativen Bestimmung, denen Umsetzungen zu Grunde liegen, sind zu bekannt, um dieselben an dieser Stelle ausfübrlicher zu erörtern; ich beschränke mich darauf, die von Lunge (Chem. Ind. 1881, 369) empfohlene Methode, bei welcher mittels $1 / 2 \mathrm{~N}$.-Ammoniaklösung im Überschusse Bicarbonat in Carbonat umgewandelt wird und die überschüssig zugesetzte Menge Ammoniaklösung nach Versetzen mit überschüssiger Chlorbaryumlösung durch Titration eines aliquoten Theiles des Filtrates bestimmt wird, $z u$ erwähnen, wie an die ähnliche, von Böckmann empfohlene $\mathrm{Me}-$ thode, wobei Ammoniak durch Natronlauge ersetzt ist und dadurch die Filtration umgangen wird, zu erinnern.
Die bekannte Trennungsmethode von Hydroxyd und Carbonat mittels Chlorbaryumlösung nach den Gleichungen:

$\mathrm{Na}_{2} \mathrm{CO}_{3}+\mathrm{BaCl}_{2}=2 \mathrm{NaCl}+\mathrm{BaCO}_{3}$ und

$2 \mathrm{NaOH}+\mathrm{Ba} \mathrm{Cl}_{2}=2 \mathrm{NaCl}+\mathrm{Ba}(\mathrm{OH})_{2}$

ergibt unter strenger Beobachtung der sich von selbst ergebenden Vorsichtsmaassregeln zwar brauchbare Resultate, welche aber an Genauigkeit ebenso wie an Bequemlichkeit und Schnelligkeit in der Ausführung der Methode hinter denen der oben angegebenen Titrationen mit Alcannin, Phenolphtalein und Bleu de Lyon weit zurückstehen.

Die Berechnung für Hydroxyd, Carbonat und Bicarbonat bei Anwendung der Titrationsmethoden ergibt sich leicht. In Lösungen, welche Hydroxyd und Carbonat enthalten, wird bei der ersten Phase der Titration eine dem Hydroxyd + der Hälfte der gebundenen Koblensāure äquivalente Menge Säure verbraucht, die zweite Phase der Titration (mit Methylorangezusatz) zeigt nur die Hälfte der gebundenen Kohlensäure an, woraus sich dann Hydroxyd aus der Differenz ergibt. Bei Mischungen von Bicarbonaten und Carbonaten ist bei der ersten Phase der Titration eine der Hälfte der Monocarbonate äquivalente Menge Titrationssäure nöthig, während die zweite Phase der Titration die Hälfte der ursprünglich zu Monocarbonat gebundenen Kohlensäure + der Gesammtmenge der Monocarbonatsäure, welche in der ursprünglichen Flüssigkeit als Bicarbonat gebunden war, anzeigt. Gesammttitrationssäureverbrauch (1. + 2. Phase) ist also äquivalent der Gesammtmonocarbonatsäure, während sich die ursprünglich als Bicarbonat gebundene Kohlensäuremenge durch Differenz aus dem Säureverbrauch der zweiten Phase minus dem der ersten Phase der Titration ergibt und durch 2 dividirt die wahre balbgebundene, d. h. die in der Flüssigkeit mit Monocarbonat zu Bicarbonat gebundene Menge Kohlensäure anzeigt.

Will man mit diesen Titrationsmethoden gelöstes Hydroxyd der alkalischen Erden neben Alkalihydroxyd bestimmen, so lässt sich dies leicht in der Weise ausführen, dass man zunächst die dem Gesamm thydroxyd äquivalente Menge Säure durch Titration unter Benutzung von Phenolphtalein oder eines der anderen empfindlichen Indicatoren feststellt und sodann in einem anderen Theile der Flüssigkeit das Hydroxyd der alkalischen Erden mit titrirter Alkalicarbonatlösung fällt und in einem aliquoten Theile des Filtrates das überschüssige Alkalicarbonat neben Alkalihydroxyd, wie oben angegeben, mit Alcannin, Bleu de Lyon oder Phenolphtalein bestimmt, wonach sich durch $\mathrm{Be}$ - 
rechnung leicht Hydroxyd der alkalischen Erde und Hydroxyd des Alkalis in der ursprünglichen Flüssıgkeit berechnen lässt.

In analoger Weise lässt sich Magnesiumcarbonat bei gleichzeitig gelöstem Alkalicarbonat durch Natriumbydroxydlösung trennen (Erhitzen der Lösung ist nothwendig), oder aber der Gehalt an Magnesiumcarbonat mit Hàmatoxylin und sodann der Gesammtcarbonatgehalt mit Methylorange feststellen, wober aber im letateren Falle, wie oben näher erörtert worden ist, ungefähr nur $2 / 3$ des vorhandenen Magnesiumcarbonates als solches angezeigt werden würde.

Von der Thatsache nun ausgehend, dass Alkalihydroxyd mit freier Kohlensäure Carbonat und Alkalicarbonat mit freier Kohlensäure Bicarbonat bildet, lassen sich auch leicht Metboden zur Bestimmung der freien Kohlensäure neben Bicarbonaten aufstellen, sofern ein gegeuüber freier Kohlensäure wie gegenüber des angewandten Carbonates oder Hydroxydes empfindlicher Indicator zugegen ist. Und hierbei eignen sich am besten Phenolphtalein und Tropäolin 000, worüber ich an der zu Anfang dieser Arbeit citirten Stelle nähere Angaben gemacht habe und auf jene verweise. Auf die Benutzung und Bedeutung der Indicatoren Hämatoxylin, Gallein, Alcannin, Gentianablau und Bleu de Lyon für die qualitative und quantitative Analyse in ungebundener Koblensäure freien Wässern bez. Flüssigkeiten babe ich ebenfalls in genannter Arbeit und zwar im III. Abschnitte derselben an der Hand einer Reıhe untersuchter Wässer hingewiesen.

\section{Über die quantitative \\ Bestimmung von Mangan, Magnesium, Zink, Kobalt und Nickel mittels der Oxalatmethode nach Prof. A. Classen.}

$$
\text { Von }
$$

\section{Dr. G. Nass.}

\section{Mangan.}

Als ich vor einiger Zeit die quantitative Bestimmung des Mangans mittels der Oxalatmethode nach Prof. Classen vornahm, konnte ich so überaus befriedigende Resultate, wie sie Herr Classen für diese $\mathrm{Me}$ thode anführt, nicht erhalten. Ja, die Bestimmungen führten zu ganz ungenugenden Zahlen, die die erlaubte Fehlergrenze ganz bedeutend überschritten.

Diese Thatsache nun bestimmte mich, die angeregte Methode genau durchzuführen und einer eingehenderen Untersuchung zu unterziehen und im Anschluss hieran auch einige Versuche über die entsprechenden Vorschläge zur quantitativen Bestimmung von Magnesium, Zink, Kobalt und Nickel anzustellen.

In seinem "Handbuch der quantitativen chemischen Analyse", IV. Auflage (1891) S. 33 bez. 28 gibt Herr Prof. Classen') zur Bestimmung des Mangans folgende Vorschrift.

Man fuge $/ u$ der 20 bis 25 ce betragendeu wassrigen neutralen Losung des Mangansalzes so viel neutrales Kaliumovalat ( $1 \mathrm{Th}$ Kaliumoxalat in $3 \mathrm{Th}$ Wasser gelost), bis der entstandene Niederschlag von Manganoxalat sich unter Bildung ron Mangankaliumoxalat wieder gelost hat, erhit/e rum Sieden, fuge nach und nach unter Umruhren so vel concentrurte Essigsaure ( 80 bis 90 proc) hinzu, bis nach dem Absitzen des Niederschlages auf weiteren Zusatz von Essigsaure keine Fallung mehı entsteht, und setze das Kochen unter stetem Umruhren noch kurze Zeit fort. Die Menge der Essigsaure soll mindestens das Volumen der zu fallenden Flussigkeit betragen. Nach etwa sechsstundigem Stehen des gut bedeckten Gefasses ber etwa $50^{\circ}$ filtrire man den Niederschlag $a b$ und wasche mit emer Mischung aus gleıchem Volumen cone Essigsaure, Alkohol und Wasser so lange aun, bis ein Tropfen des Filtrats, auf dem Platinblech verdampft, kemen Ruckstand hinterlasst. Das Manganoxalat fuhre man dann durch Gluhen im Platuntiegel in Manganoxydoxydul uber. Bel micht genugendem Auswaschen enthalt der Niederschlag Kahumovalat, das beim Gluhen Kalumcarbonat gibt, welches, nach Prufung mit Lackmuspapier, durch Digerien mit Wasser auf dem Sand- oder Wasserbad zu losen und von dem Ruckstand durch Filtriren und Auswaschen mit heissem Wasser zu entfernen ist.

Als Beleganalysen fügt Herr Classen ( $Z$. anal. 16, 316) die folgenden an:

$20 \mathrm{cc}$ einer Manganchlorurlosung gaben um Mittel aus 2 Bestimmungen 0,3375 g Schwefelmangan.

20 cc derselben lieferten 0,2975

20 - $\quad$ - $\quad$ - 0,2965

20 - - $\quad$ - 0,2965

im Mittel also 0,2968 g Manganoxydoxydul. $0,3775 \mathrm{~g} \mathrm{MnS}$ entsp. $0,2131 \mathrm{Mn}=1,0655 \mathrm{~g} \mathrm{Mn} \mathrm{f.100 \textrm {ce }}$ $0,2965 \mathrm{~g} \mathrm{Mn}_{3} \mathrm{O}_{4}-0,2137 \mathrm{Mn}=1,0665 \mathrm{~g}$ - 100 -

Ferner wird angegeben (das. 317), dass die Anwesenheit von Chlorammonium und Chlorkalium die Genauigkeit der Methode nachtheilig beeinflusst.

In der mir zu Gebote stehenden Litteratur fand ich nur zwei Veröffentlichungen über diese Methode. Zunächst eine Broschüre von Dr. F. Kessler: „Uber die Bestimmung des Mangans, besonders in Eisen-Manganlegirungen". Dieselbe befasst sich hauptsächlich nur mit der Trennung von Mangan und Eisen und führt als Nach-

1) S auch Z. anal. 16 S. 315 Ber. deutsch. G X. 2. S. 1315 . 